\title{
Resultados quirúrgicos de histerectomía radical laparoscópica en pacientes con cáncer de cérvix en etapa temprana: experiencia inicial en Instituto de Cáncer
}

\author{
Surgical outcomes of laparoscopic radical hysterectomy for patients with early cervical \\ cancer: Initial experience in Cancer Institute
}

\author{
David Isla-Ortiz"*, Rosa A. Salcedo-Hernández', Alberto M. León-Takahashi', Fabiola Estrada-Rivera³, \\ Salim A. Barquet-Muñoz y Nancy Reynoso-Noverón ${ }^{2}$ \\ ${ }^{1}$ Departamento de Ginecología Oncológica; ${ }^{2}$ Departamento de Epidemiología. Instituto Nacional de Cancerología México; ${ }^{3}$ Departamento de \\ Ginecología Oncológica, Instituto Nacional de Perinatología México. Ciudad de México, México
}

\begin{abstract}
Resumen
Antecedentes: La histerectomía radical abierta con linfadenectomía pélvica bilateral es el tratamiento estándar para el cáncer de cérvix uterino (CACU) en etapas temprana (1A2-1B1); la histerectomía radical por laparoscopia (HRL) es una opción segura y viable. Objetivo: Evaluar la seguridad y la factibilidad de la HRL en un centro hospitalario de atención de cáncer. Método: Estudio retrospectivo que incluyó 17 pacientes con CACU en etapa temprana intervenidas con HRL entre abril de 2013 y noviembre de 2016 en el Instituto Nacional de Cancerología de México. Resultados: Las 17 pacientes se encontraban en etapa clínica IB1, en 10 (58.8\%) fue subtipo epidermoide, en 4 (23.5\%) adenocarcinoma y en 3 (17.6\%) adenoescamoso. La media de edad fue de $42 \pm 8$ años. El tamaño del tumor fue de $2.3 \pm 0.9 \mathrm{~cm}$, y en el $94.1 \%$ los márgenes quirúrgicos estaban libres de enfermedad. El promedio de tiempo operatorio fue de $341 \pm 65$ minutos, con una pérdida sanguínea de $107 \pm 64 \mathrm{ml}$, no requirieron trasfusión sanguínea y no hubo conversión a cirugía abierta. La media de estancia hospitalaria fue de 2.7 días (rango: 2-7 días). No se presentaron complicaciones intraoperatorias ni posoperatorias. Conclusiones: La $H R L$ es una alternativa segura y confiable para el tratamiento del CACU en etapa temprana.
\end{abstract}

PALABRAS CLAVE: Histerectomía radical laparoscópica. Etapa temprana. Cáncer de cérvix.

\begin{abstract}
Background: Open radical hysterectomy with bilateral pelvic lymphadenectomy is the standard treatment in early stages (1A2-1B1) of uterine cervical cancer (UCC); laparoscopic radical hysterectomy (LRH) is a safe and viable option. Objective: To evaluate the safety and feasibility of $L R H$ in a hospital cancer care center. Method: Retrospective study that included the first 17 patients with UCC in an early stage operated with LRH in the period from April 2013 to November 2016 at the National Cancer Institute of Mexico. Results: The 17 patients were stage IB1 clinical, of which 10 (58.8\%) was epidermoid subtype, 4 (23.5\%) adenocarcinoma and $3(17.6 \%)$ adenoescamoso. The mean age was $42 \pm 8$ years. The tumor size was $2.3 \pm 0.9 \mathrm{~cm}$, and in $94.1 \%$ the surgical margins were free of disease. The average operative time was $341 \pm 65$ minutes and blood loss of $107 \pm 64 \mathrm{ml}$, no patient required blood transfusion and there was no case of conversion to open surgery. The average length of hospital stay was 2.7 days (range: 2-7 days). There were no intraope-
\end{abstract}

\author{
Correspondencia: \\ *David Isla-Ortiz \\ Av. San Fernando, 22 \\ Col. Sección XVI, Del. Tlalpan \\ C.P. 14080, Ciudad de México, México \\ E-mail: islasurgery@ hotmail.com
}

Fecha de recepción: 08-02-2018

Fecha de aceptación: 02-04-2018

DOI: 10.24875/CIRU.M18000035
Cir Cir. 2018;86:220-227

Contents available at PubMed www.cirugiaycirujanos.com 
rative or postoperative complications. Conclusions: $L R H$ is a safe and reliable alternative for the treatment of early stage UCC.

KEY WORDS: Radical hysterectomy. Early stage. Cervical cancer.

\section{Introducción}

El cáncer de cérvix es el segundo cáncer más común en mujeres en los países en desarrollo y el séptimo más común en los países desarrollados ${ }^{1,2}$. En México, un país en desarrollo, continúa siendo un problema de salud pública, ya que además de ser el segundo cáncer en incidencia y mortalidad, aún se sigue presentando en etapas localmente avanzadas ${ }^{3}$. Sin embargo, con el uso eficaz de la detección oportuna de cáncer de cérvix, muchas mujeres serán diagnosticadas en edad fértil y en una etapa temprana $(\mathrm{IA} 2 \text { y IB1 })^{4}$, cuyo tratamiento estándar es la histerectomía radical abierta con linfadenectomía pélvica bilateral $^{5}$. El abordaje laparoscópico descrito por Canis, et al. ${ }^{6}$ y Nezhat, et al. ${ }^{7}$ ha mostrado su seguridad y factibilidad en múltiples estudios, con resultados oncológicos similares a los observados por la vía abierta, pero con una menor tasa de morbilidad quirúrgica, menor sangrado, estancia hospitalaria más corta y reincorporación más temprana a las actividades cotidianas. Steed, et al. ${ }^{8}$ compararon, de 1996 a 2003, la morbilidad perioperatoria de 71 pacientes tratadas con histerectomía radical por laparoscopia (HRL) en comparación con 205 pacientes tratadas con histerectomía radical abierta, reportando pérdidas de sangre de 300 y $500 \mathrm{ml}$, y un tiempo operatorio de 3.5 y 2.5 horas, respectivamente. Las complicaciones intraoperatorias en la laparoscopia incluyeron cistotomía, lesión de uréteres y lesión intestinal, por lo que se concluye que la $\mathrm{HRL}$ es un procedimiento seguro, con baja morbilidad general y bajas tasas de complicacio$n^{8}{ }^{8}$. La HRL se realiza en países desarrollados y en algunos países en vías de desarrollo. Una de las preocupaciones con respecto a los estudios prospectivos y multicéntricos de cirugía ginecológica, en particular aquellos que involucran el uso de la cirugía laparoscópica, es el tipo y la calidad de la tecnología con que se cuenta en los países en vías de desarrollo, que limitarían la realización de este abordaje, pero comparar una serie de casos de la implementación de este abordaje con las experiencias de países desarrollados permitirá evaluar la factibilidad y la seguridad de la $H R L$ con linfadenectomía pélvica bilateral. Por lo tanto, el objetivo de este estudio es mostrar la experiencia inicial de la implementación de esta cirugía en el Instituto Nacional de Cancerología de México para el tratamiento del cáncer de cérvix en etapas tempranas, así como comparar los resultados patológicos, quirúrgicos y de las complicaciones con los reportados en la literatura.

\section{Método}

Se realizó una revisión retrospectiva de todas las pacientes con diagnóstico de cáncer de cérvix en etapa temprana (IA2-IB1, de acuerdo con la Federación Internacional de Ginecología y Obstetricia [FIGO]) que se sometieron a HRL en el periodo de abril de 2013 a noviembre de 2016 en el Instituto Nacional de Cancerología de México. Se incluyeron las pacientes con histologías epidermoide, adenocarcinoma o adenoescamoso, y se excluyeron otras histologías.

Se recolectaron los siguientes datos: edad, etapa clínica, histología, duración de la cirugía, sangrado, transfusión sanguínea, complicaciones transoperatorias y posoperatorias, conversión a cirugía abierta, días de estancia hospitalaria y morbilidad durante los primeros 30 días del posoperatorio. También se obtuvieron la evaluación de la radicalidad del procedimiento, la cual fue definida como la identificación de todas las estructuras anatómicas y corte a nivel de los límites descritos por Querleu y Morrow ${ }^{9}$, y los reportes de los hallazgos de patología, como tamaño tumoral, infiltración al estroma cervical, permeación linfovascular, número de ganglios, evaluación de los parametrios y márgenes quirúrgicos de resección.

\section{Técnica quirúrgica}

La técnica quirúrgica utilizada es la siguiente: bajo anestesia general, las pacientes son colocadas en posición de Trendelenburg y se inicia con la colocación de un movilizador uterino tipo VCare $^{\circledR}$ Uterine Manipulator/Elevator (ConMed Endosurgery, Utica, NY) en el útero; posteriormente, a través de una incisión supraumbilical de $10 \mathrm{~mm}$ se introduce un trocar Endopath Bladeless Trocarendopath Optiview Optical Obturator (Ethicon) hasta la cavidad abdominal y se insufla con $\mathrm{CO}_{2}$ hasta una presión de $15 \mathrm{mmHg}$. Se utilizan cuatro trocares de $5 \mathrm{~mm}$; dos se colocan a derecha e 
izquierda de los cuadrantes inferiores, y los otros dos a derecha e izquierda de los flancos a nivel de la cicatriz umbilical. Se explora la cavidad abdominal, se realiza la apertura con corte monopolar del ligamento ancho en un triángulo formado por el ligamento redondo, el músculo psoas y el ligamento infundíbulo-pélvico, desplazando el corte paralelo a este último; esto se logra colocando el útero en contratracción al corte y el ligamento redondo traccionado hacia arriba, disecando los espacios retroperitoneales paravesicales y pararrectales, y posteriormente el espacio recto-vaginal y la disección del tejido vésico-vaginal. Después se seccionan los ligamentos redondos, el ligamento infundíbulo-pélvico (con excepción de los casos en que se preservan los ovarios). Se exploran los ganglios pélvicos para descartar evidencia de malignidad y así posteriormente proceder a identificar las arterias uterinas, que se coagulan y cortan a nivel de la emergencia de la arteria hipogástrica con un coagulador bipolar LigaSure ${ }^{\circledR}$ laparoscópico (5 mm blunt tip, Medtronic). Los uréteres son liberados del tejido adyacente peritoneal y del parametrio lateral, dorsal y ventral. La vejiga es disecada para exponer los pilares vesicales, los cuales son coagulados y resecados de la pared posterior vesical. La vagina se secciona $1 \mathrm{~cm}$ por debajo del cérvix con gancho monopolar (Karl Storz), y se extrae la pieza quirúrgica por vía vaginal. La linfadenectomía pélvica bilateral se realiza antes de la histerectomía cuando hay sospecha de metástasis ganglionar por imagen 0 durante la cirugía, disecando el tejido linfático de los vasos iliacos comunes, externos y de la fosa obturatriz (Fig. 1), aplicando los límites quirúrgicos convencionales. El tejido linfático se extrae por vía vaginal y posteriormente la cúpula vaginal se sutura en dos planos con un súrgete continuo utilizando poliglactina $\left(\right.$ Vicryl $\left.{ }^{\circledR}\right)$ 2-0. El útero (Figs. 2 y 3) y el tejido son evaluados por patólogos de nuestro Instituto.

\section{Análisis estadístico}

Se determinaron medidas de tendencia central y dispersión para cada una de las variables exploradas en el estudio, presentando media o mediana, desviación estándar o rango, o porcentaje, según correspondiera. Los parámetros estadísticos se obtuvieron mediante el programa SPSS V 20.0 (IBM Corporation).

\section{Resultados}

Durante el periodo de estudio, comprendido entre abril de 2013 y noviembre de 2016, 17 pacientes fueron

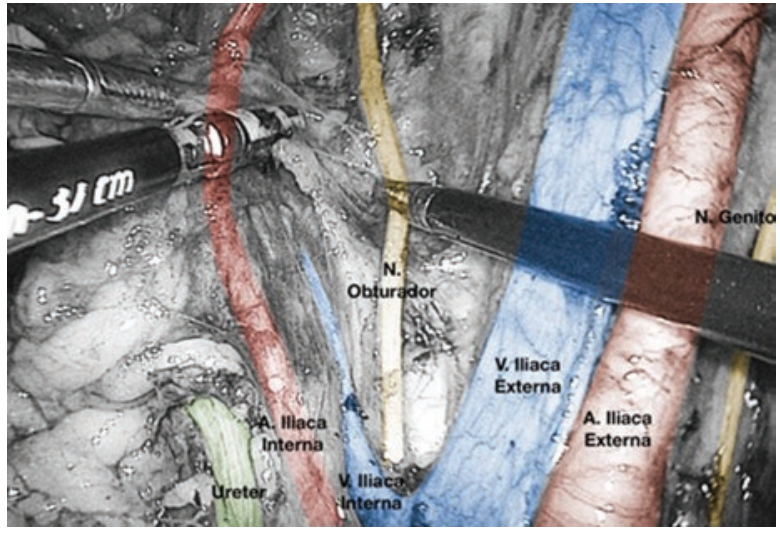

Figura 1. Estructuras anatómicas identificadas durante la linfadenectomía pélvica (lado derecho).

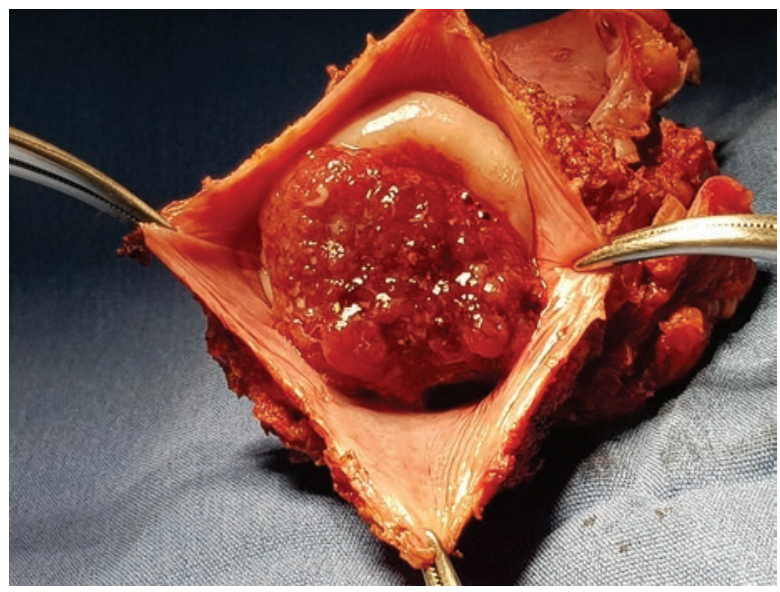

Figura 2. Pieza de patología de histerectomía radical, mostrando la relación del tumor con el cérvix no afectado y los márgenes vaginales.

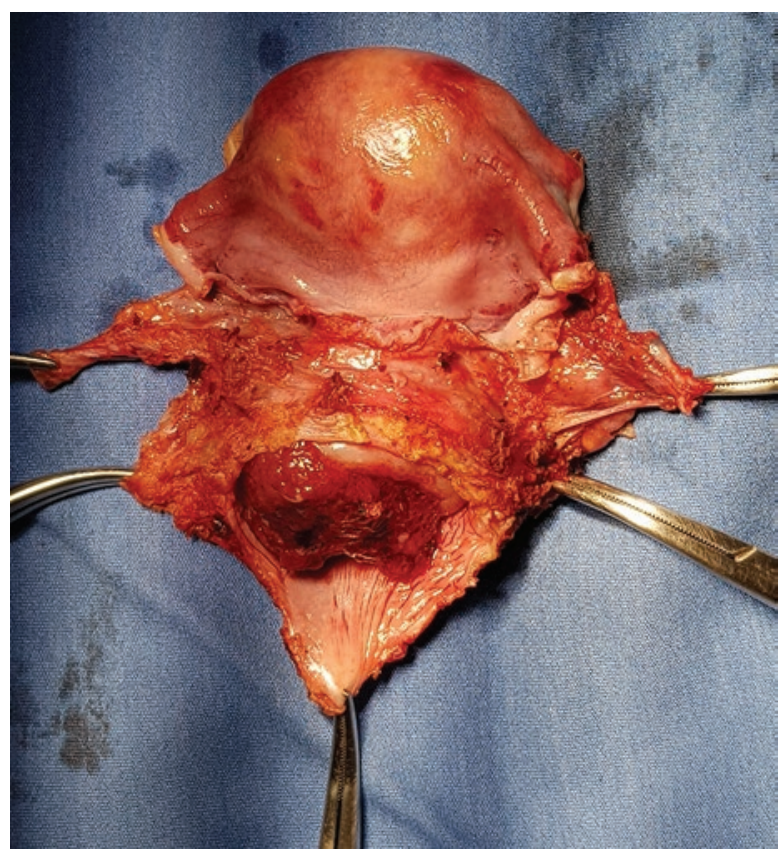

Figura 3. Pieza de patología de histerectomía radical, visualizando parametrios laterales y arteria uterina. 
tratadas con HRL y linfadenectomía pélvica bilateral. La edad promedio fue de 42 años (rango: 32-61 años), el índice de masa corporal fue de 27.7 (rango: 19.235.7) y todos los casos fueron clasificados como etapa clínica 1B1 (Tabla 1). Se realizaron 13 histerectomías radicales tipo $\mathrm{B}$ y cuatro tipo $\mathrm{C} 1$ (clasificación de Querleu-Monrrow) ${ }^{9}$. El tiempo quirúrgico promedio fue de $341 \pm 65$ minutos (media \pm desviación estándar), el promedio de pérdida sanguínea fue de $107 \mathrm{ml}$ (rango: 20-250) y ninguna requirió transfusión sanguínea. No se presentaron complicaciones transoperatorias ni conversión a abordaje convencional. Durante el posquirúrgico, la estancia hospitalaria en promedio fue de 2.7 días (rango: 2-7); solo un caso se mantuvo por 7 días debido a síndrome febril por infección de vías urinarias que remitió con tratamiento antibiótico, y ningún caso requirió terapia intensiva (Tabla 2). El reporte histopatológico confirmó 10 casos (58.8\%) de carcinoma epidermoide, 4 (23.5\%) de adenocarcinoma y $3(17.6 \%)$ de carcinoma adenoescamoso; el tamaño tumoral promedio fue de $2.3 \mathrm{~cm}$ (rango: $0.5-4$ ), el grado de invasión cervical promedio fue de 1/3, $9(52.9 \%)$ presentaron permeación linfovascular y 1 (5.8\%) borde quirúrgico positivo. El número promedio de ganglios obtenidos en la linfadenectomía pélvica bilateral fue de 16 (8 de la derecha y 8 de la izquierda) (Tabla 1). El $47 \%$ de las pacientes recibió tratamiento adyuvante por riesgo de recurrencia; la mitad $(23.53 \%)$ recibieron solo radioterapia (teleterapia 50 Gy a la pelvis) y braquiterapia (24 Gy a tasa alta) por encontrarse en riesgo intermedio, y la otra mitad (23.53\%) tuvieron como tratamiento adyuvante quimioterapia y radioterapia (teleterapia y braquiterapia) por presentar factores de alto riesgo de recurrencia (dos por ganglios pélvicos positivos y una por borde quirúrgico positivo). En contraste, el 52.94\% no recibió ningún tratamiento adyuvante (Tabla 3). Después de una mediana de 13 meses (rango: 1-25) no se reportaron recurrencias ni defunciones; solo a una paciente se le realizó otra operación durante la braquiterapia al presentar apertura de la cúpula vaginal, en la que se realizó cierre primario vaginal sin complicaciones.

La comparación de nuestros hallazgos con los principales resultados oncológicos, de seguridad y de morbilidad previamente encontrados en la literatura se muestra en la tabla 4.

\section{Discusión}

El uso del abordaje laparoscópico en el tratamiento del cáncer de cérvix ofrece los beneficios de una
Tabla 1. Características clínico-patológicas $(n=17)$

\begin{tabular}{lc}
\hline Edad (años) & Mediana: 40 (rango: 32-61) \\
\hline Índice de masa corporal $\left(\mathrm{kg} / \mathrm{m}^{2}\right)$ & 27.7 (rango: 19.2-35.7) \\
Etapa clínica 1B1 & $17(100 \%)$ \\
Tamaño del tumor & $2.3 \pm 0.9$ \\
Subtipo histológico & \\
Epidermoide & $10(58.8 \%)$ \\
Adenocarcinoma & $4(23.5 \%)$ \\
Adenoescamoso & $3(17.6 \%)$ \\
Reporte de patología & \\
Parametrios & \\
Negativo & $17(100 \%)$ \\
Márgenes quirúrgicos & \\
Positivos & $1(5.8 \%)$ \\
Negativos & $16(94.1 \%)$ \\
Infiltración del estroma & \\
1/3 & \\
$2 / 3$ & $9(52.9 \%)$ \\
$3 / 3$ & $4(23.5 \%)$ \\
Permeación linfovascular & $4(23.5 \%)$ \\
Positivo & \\
Negativo & $9(52.9 \%)$ \\
\hline Los datos son reportados en medias ( \pm desviación estándar) o n (\%). & \\
&
\end{tabular}

Tabla 2. Datos perioperatorios $(n=17)$

\begin{tabular}{lc}
\hline $\begin{array}{l}\text { Promedio de ganglios obtenidos en la } \\
\text { linfadenectomía }\end{array}$ & $16 \pm 7$ \\
Ganglios positivos & $0(\%)$ \\
Pérdida sanguínea $(\mathrm{ml})$ & $107( \pm 64)$ \\
Transfusiones & 0 \\
Tiempo quirúrgico (min) & $341( \pm 65)$ \\
Duración de estancia hospitalaria & $12(70.5 \%)$ \\
2 días & $1(5.8 \%)$ \\
3 días & $3(17.6 \%)$ \\
4 días & $1(5.8 \%)$ \\
7 días & $0(0 \%)$ \\
Defunciones & \\
\hline
\end{tabular}

Los datos son reportados en medias ( \pm desviación estándar) o n (\%).

Tabla 3. Seguimiento y tratamiento adyuvante $(n=17)$

\begin{tabular}{lc}
\hline Seguimiento (meses) & $14( \pm 8)$ \\
Tratamiento adyuvante & \\
$\quad$ Ninguno & $9(52.94 \%)$ \\
RT y BT (por riesgo intermedio de recurrencia) & $4(23.53 \%)$ \\
QT/RT y BT (por riesgo alto de recurrencia) & $4(23.53 \%)$ \\
Recurrencias & $0(0 \%)$ \\
Defunciones & $0(0 \%)$ \\
\hline
\end{tabular}

Los datos son reportados en medias ( \pm desviación estándar) o n (\%).

BT: braquiterapia; QT: quimioterapia; RT: radioterapia. 
menor pérdida sanguínea, una corta estancia hospitalaria en comparación con la laparotomía, pequeñas incisiones, menor dolor posoperatorio, recuperación más rápida, mejor calidad de vida durante el posoperatorio, y mejores resultados cosméticos, siendo un procedimiento factible y seguro $0^{8,10-13}$.

La HRL otorga como beneficio la disminución de pérdida sanguínea con respecto a la histerectomía radical abierta, como se ha reportado en los estudios de Puntambekar, et al..$^{14} \mathrm{y}$ Kong, et al..$^{15}$, así como en el estudio aleatorizado de Naik, et al. ${ }^{10}$, en el que se comparó la histerectomía radical por abordaje laparoscópico versus abdominal, demostrando ventajas significativas del abordaje laparoscópico sobre el abdominal, específicamente una menor pérdida sanguínea (400 versus $1000 \mathrm{ml}$ ), una menor duración de la cateterización vesical (4 versus 21 días), menos necesidad de opiáceos durante las primeras 36 horas del posoperatorio (30 versus $53 \mathrm{mg}$ ) y una estancia hospitalaria más corta (5 versus 7 días), aunque el abordaje laparoscópico tomó más tiempo en realizarse (4 versus 2.3 horas).

En nuestro estudio, la pérdida sanguínea fue de $107 \mathrm{ml}$, y los días de estancia hospitalaria promedio fueron 2.7, aún menos que lo reportado por Naik, et al..$^{10}$ para cirugía laparoscópica; sin embargo, el tiempo de cirugía superó en promedio 1.5 horas lo reportado previamente.

Con respecto a los tiempos operatorios, en el estudio de Ramírez, et al. ${ }^{16}$ el tiempo operatorio promedio reportado fue de 332 minutos, similar al tiempo operatorio promedio de 341 minutos de nuestro estudio; sin embargo, el tiempo reportado por Puntambekar, et al. ${ }^{14}$ fue de 80 minutos en promedio, que es el menor tiempo registrado de HRL. Asimismo, reportaron que la estancia hospitalaria en promedio fue de 1 día, valores significativamente menores que los controles históricos sometidos a histerectomía radical abierta. La cantidad de ganglios extirpados en nuestro estudio fue de 16, número similar al reportado por Ramírez, et al. ${ }^{16}$, con 13 ganglios, y por $\mathrm{Li}$, et al. ${ }^{17}$, con 21.28 ganglios.

Las limitaciones de la laparoscopia convencional incluyen la visualización bidimensional, una plataforma de cámara inestable, grados de movimiento limitados utilizando un instrumento dentro del cuerpo, así como la dificultad ergonómica, la amplificación del temblor fino y la dificultad para realizar procedimientos quirúrgicos complejos en algunas circunstancias, tales como la presencia de adherencias densas en el abdomen?.
Una de las principales complicaciones de la histerectomía radical es la disfunción vesical posquirúrgica. En el estudio de Ramírez, et al. ${ }^{16}$ ninguna de las pacientes sufrió disfunción vesical. Los informes en la literatura de las tasas de disfunción de vesical son de hasta el 20\% después de la histerectomía abdominal radical abierta. En el estudio de Li, et al. ${ }^{17}$ se encontró una disminución significativa en la tasa de retención urinaria en las pacientes sometidas a HRL en comparación con la histerectomía abdominal abierta, y en el estudio de Yang, et al. ${ }^{18}$ el $37.25 \%$ de las pacientes tenía disfunción vesical. La limitación en la radicalidad de la cirugía puede reducir al mínimo esta morbilidad. Otras de las complicaciones publicadas para la HRL son lesión uretral, fístulas uretrales y fistula vésico-vaginal; lesiones similares a las que se presentan en la histerectomía radical abierta. En cuanto a la lesión uretral, en el estudio de Kong, et al..$^{15}$ se ha reportado una tasa de incidencia del 1.9-2.3\%; esta alta tasa puede deberse a que en ese estudio se incluyeron pacientes con un diámetro tumoral $\geq 3 \mathrm{~cm}$, ya que en la mayoría de los estudios las pacientes incluidas se encuentran en estadio FIGO IA1 a IB1. Las complicaciones intraoperatorias en una serie de 404 pacientes a las que se realizó HRL ocurrieron en siete pacientes, y la tasa de conversión a cirugía abierta fue del $0.50 \%{ }^{18}$.

La curva de aprendizaje de la laparoscopia convencional ha sido un problema para los nuevos cirujanos. Wattiez, et al..$^{19}$ publicaron un estudio comparativo retrospectivo sobre la curva de aprendizaje de la HRL de 1647 casos, y concluyeron que los tiempos de funcionamiento y las principales complicaciones disminuyeron significativamente después de un entrenamiento adecuado. Serna, et al..$^{20}$ identificaron que en promedio es necesaria la práctica de $50 \mathrm{HRL}$ para que un cirujano pueda realizar la intervención en un tiempo promedio menor de 90 minutos; sin embargo, tras $200 \mathrm{HRL}$ se llega a una llanura, estableciéndose tiempos de cirugía de alrededor de 83 minutos. Así mismo, la práctica permite que el cirujano aumente su destreza y disminuya paulatinamente la pérdida sanguínea, presentándose en las primeras 200 cirugías una pérdida de $75.4 \mathrm{ml}$ y disminuyendo a $52.6 \mathrm{ml}$ después de 420 laparoscopias. Para lograr un porcentaje de laparoconversión del 3\% o menos son necesarias $50 \mathrm{HRL}$, disminuyendo paulatinamente conforme se realizan más histerectomías. Las complicaciones menores después de tener la experiencia de $200 \mathrm{HRL}$ son del $6 \%$, y este porcentaje se mantiene estable aun después de realizar más de 600 intervenciones quirúrgicas. 

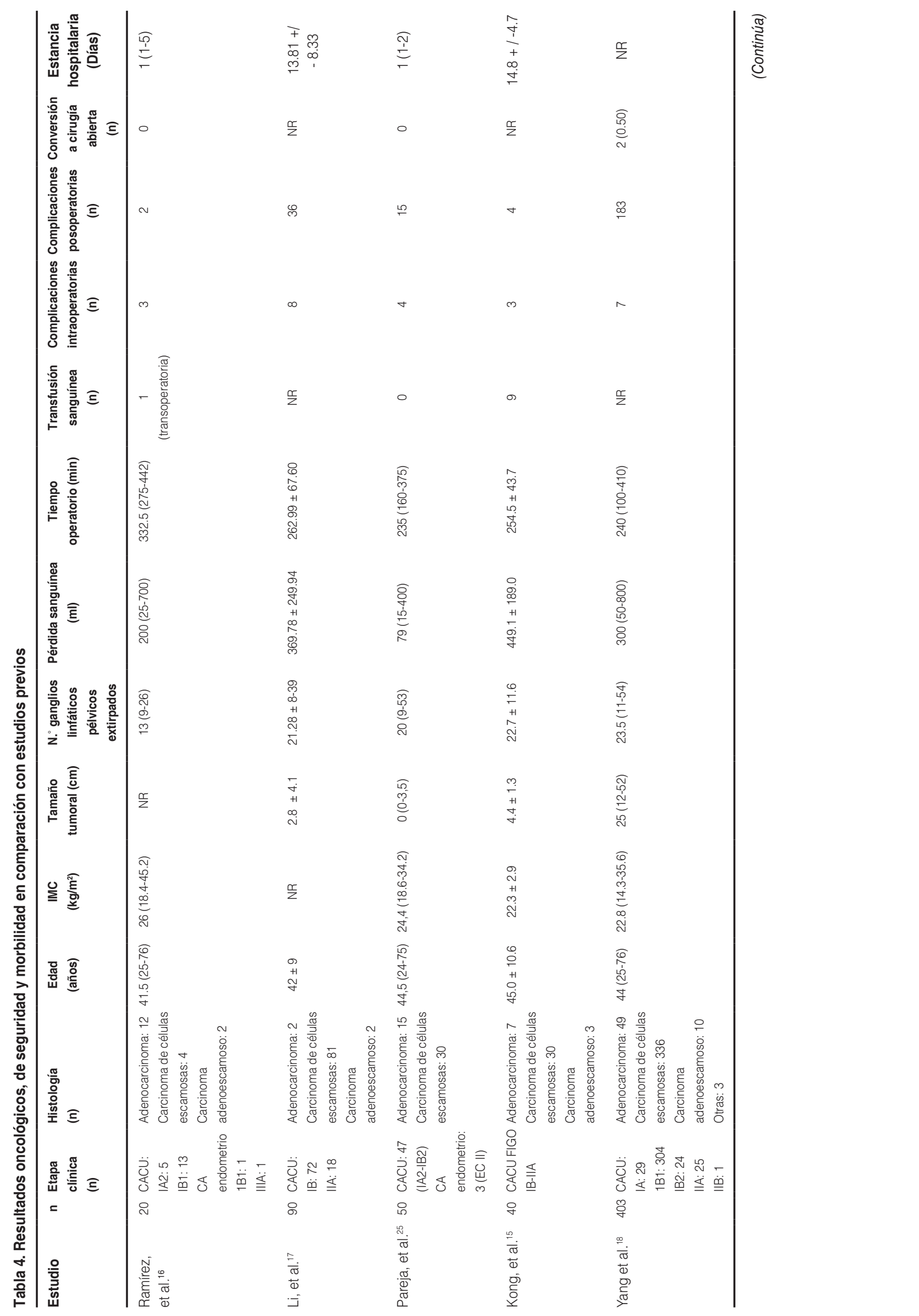


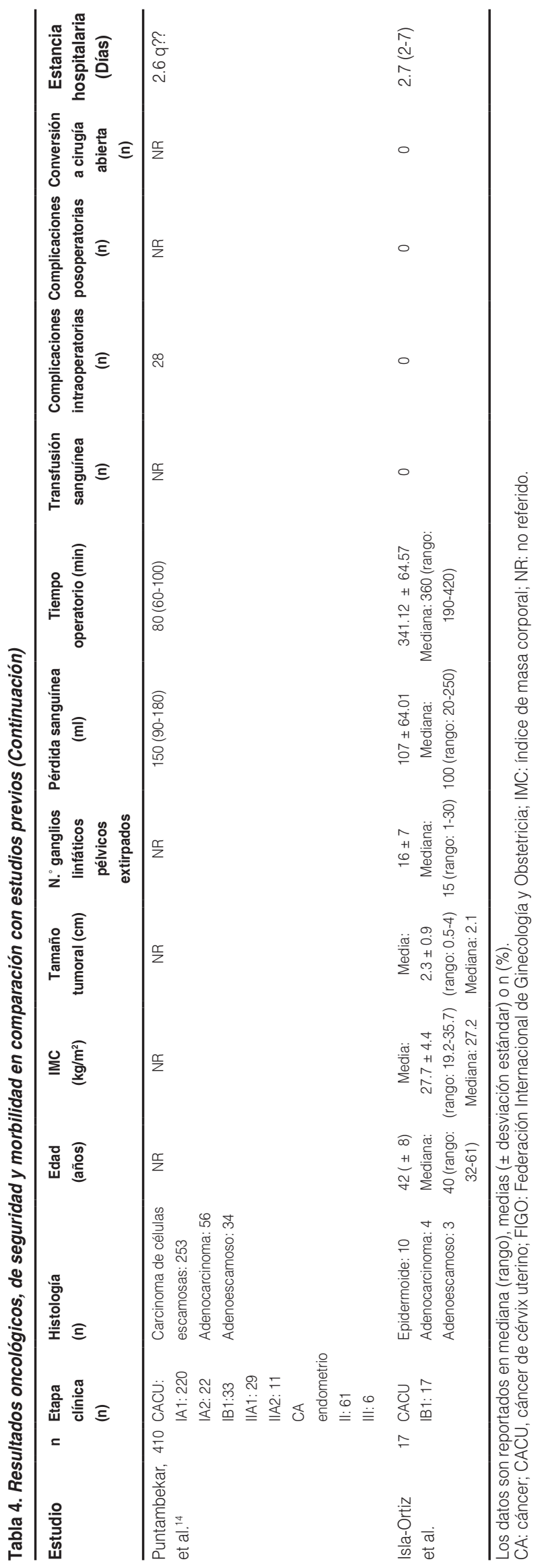

Perino, et al. ${ }^{21}$ compararon los tiempos de cirugía entre histerectomías abdominales y HRL, e identificaron que en la histerectomía abdominal después de 15 cirugías el tiempo se estabiliza en 87 minutos, pero en las laparoscópicas las primeras 15 intervenciones rondan los 129 minutos y entre la 16 y la 51 se llega a disminuir el tiempo hasta 93 minutos.

En el estudio realizado en Finlandia por Brummer, et al. ${ }^{22}$ notaron que conforme aumenta el número de cirugías laparoscópicas se reduce la tasa de complicaciones mayores de manera significativa, siendo necesarios casi 7 años para logar que la curva de aprendizaje llegue a su meseta. De manera puntual, Mäkinen, et al. ${ }^{23}$ mencionan que son necesarias 30 intervenciones para que se reduzcan las lesiones uretrales y vesicales.

De acuerdo con la curva de aprendizaje en la práctica quirúrgica propuesta por Hopper, et al. ${ }^{24}$, se debe llegar al estándar aceptable después de 50 intervenciones laparoscópicas, siendo el cirujano capaz de controlar el tiempo y las complicaciones mayores. El máximo nivel de destreza y el menor porcentaje de laparoconversión se alcanzan a las 100 intervenciones, y después de 150 cirugías las complicaciones totales y menores deberán encontrarse por debajo del 10\%.

La extensión de la resección radical que puede lograrse mediante el abordaje laparoscópico es controvertida. Estudios comparativos y observacionales han reportado que no existe diferencia en la extensión de la resección entre los abordajes laparoscópico y abdominal, pero existen estudios aleatorizados que demuestran que las pacientes en el grupo de laparoscopia tuvieron una disminución en la extensión del tejido resecado en la cúpula vaginal $(1.3$ vs. $2.2 \mathrm{~cm})$, el ligamento cardinal $(1.3 \mathrm{vs} .2 .8 \mathrm{~cm})$ y el ligamento uterosacro $(1.5 \text { vs. } 4.7 \mathrm{~cm})^{8,10,12}$.

El estudio de Pareja, et al. ${ }^{25}$ sugiere que las HRL se pueden llevar a cabo de manera similar en los países desarrollados, como los EE.UU., y en los países en vías de desarrollo, como Colombia, con resultados similares en cuanto a la pérdida de sangre durante la intervención, los tiempos operatorios y los tiempos de estancia hospitalaria posteriores a la laparoscopia.

Actualmente se está corriendo el ensayo clínico LACC (Laparoscopic Approach To Cervical Carcino$m a)^{26}$, un estudio internacional, multicéntrico y aleatorizado, que tiene como objetivo comparar la supervivencia libre de enfermedad en pacientes que se someten a una HRL o a una histerectomía radical abierta para el cáncer cervical en etapas clínicas IA1 y IB1 con permeación linfovascular, con histologías 
de adenocarcinoma, células escamosas o carcinoma adenoescamoso. En México se inició el reclutamiento de pacientes en julio de 2015.

\section{Conclusiones}

Las ventajas del abordaje laparoscópico son la disminución en el tiempo quirúrgico, la menor pérdida sanguínea y la estancia hospitalaria más corta. Nuestros resultados muestran que la calidad de la técnica quirúrgica de la $H R L$ en nuestra institución es comparable con lo reportado en la literatura mundial. Estos hallazgos apoyan la necesidad de conducir estudios quirúrgicos, multicéntricos e internacionales que incluyan países en vías de desarrollo.

\section{Conflicto de intereses}

Los autores declaran no tener ningún conflicto de intereses.

\section{Bibliografía}

1. Arbyn M, Autier P, Ferlay J. Burden of cervical cancer in the 27 member states of the European Union: estimates for 2004. Ann Oncol. 2007; 18:1423-5.

2. Ferlay J, Soerjomataram I, Dikshit R, Eser S, Mathers C, Rebelo M, et al Cancer incidence and mortality worldwide: sources, methods and major patterns in GLOBOCAN 2012. Int J Cancer. 2015;136:E359-86.

3. World Health Organization. Comprehensive cervical cancer control: a guide to essential practice. $2^{\text {nd }}$ ed. Geneva: WHO; 2014.

4. Díaz JP, Sonoda Y, Leitao MM, Zivanovic O, Brown CL, Chi DS, et al. Oncologic outcome of fertility-sparing radical trachelectomy versus radical hysterectomy for stage IB1 cervical carcinoma. Gynecol Oncol. 2008; 111:255-60.

5. Koh WJ, Greer BE, Abu-Rustum NR, Apte SM, Campos SM, Cho KR, et al. Cervical cancer, Version 2.2015. J Natl Compr Canc Netw. 2015; 13:395-404; quiz 404

6. Canis M, Mage G, Wattiez A, Pouly JL, Manhes H, Bruhat MA. [Does endoscopic surgery have a role in radical surgery of cancer of the cervix uteri?]. J Gynecol Obstet Biol Reprod (Paris). 1990;19:921.

7. Nezhat CR, Burrell MO, Nezhat FR, Benigno BB, Welander CE. Laparoscopic radical hysterectomy with paraaortic and pelvic node dissection. Am J Obstet Gynecol. 1992;166:864-5.

8. Steed H, Rosen B, Murphy J, Laframboise S, De Petrillo D, Covens A. A comparison of laparoscopic-assisted radical vaginal hysterectomy and radical abdominal hysterectomy in the treatment of cervical cancer. Gynecol Oncol. 2004;93:588-93.

9. Querleu D, Morrow CP. Classification of radical hysterectomy. Lancet Oncol. 2008;9:297-303.
10. Naik R, Jackson KS, Lopes A, Cross P, Henry JA. Laparoscopic assisted radical vaginal hysterectomy versus radical abdominal hysterectomy - a randomised phase II trial: perioperative outcomes and surgicopathological measurements. BJOG. 2010;117:746-51.

11. Malur S, Possover M, Michels W, Schneider A. Laparoscopic-assisted vaginal versus abdominal surgery in patients with endometrial cancer - a prospective randomized trial. Gynecol Oncol. 2001;80:239-44.

12. Hertel H, Kohler $C$, Michels W, Possover M, Tozzi R, Schneider A. Laparoscopic-assisted radical vaginal hysterectomy (LARVH): prospective evaluation of 200 patients with cervical cancer. Gynecol Oncol. 2003; 90:505-11.

13. Morgan DJ, Hunter DC, McCracken G, McClelland HR, Price JH, Dobbs SP. Is laparoscopically assisted radical vaginal hysterectomy for cervical carcinoma safe? A case control study with follow up. BJOG. 2007; 114:537-42.

14. Puntambekar SP, Sugoor D, Joshi G, Puntambekar SP, Kumbhare S, Sharma V, et al. Single institutional experience of 410 cases of type B \& type C (Querleu Morrow classification) laparoscopic radical hysterectomy. J Minim Invasive Gynecol. 2015;22(6S):S91-2.

15. Kong TW, Chang SJ, Lee J, Paek J, Ryu HS. Comparison of laparoscopic versus abdominal radical hysterectomy for FIGO stage IB and IIA cervical cancer with tumor diameter of $3 \mathrm{~cm}$ or greater. Int J Gynecol Cancer. 2014;24:280-8.

16. Ramírez PT, Slomovitz BM, Soliman PT, Coleman RL, Levenback C. Total laparoscopic radical hysterectomy and lymphadenectomy: the M.D. Anderson Cancer Center experience. Gynecol Oncol. 2006;102:252-5.

17. Li G, Yan X, Shang H, Wang G, Chen L, Han Y. A comparison of laparoscopic radical hysterectomy and pelvic lymphadenectomy and laparotomy in the treatment of Ib-lla cervical cancer. Gynecol Oncol. 2007; 105:176-80.

18. Yang L, Cai J, Dong W, Shen Y, Xiong Z, Wang H, et al. Laparoscopic radical hysterectomy and pelvic lymphadenectomy can be routinely used for treatment of early-stage cervical cancer: a single-institute experience with 404 patients. J Minim Invasive Gynecol. 2015;22:199-204.

19. Wattiez A, Soriano D, Cohen SB, Nervo P, Canis M, Botchorishvili R, et al. The learning curve of total laparoscopic hysterectomy: comparative analysis of 1647 cases. J Am Assoc Gynecol Laparosc. 2002;9:339-45.

20. Serna E, Riaño G, Almanza L, De los Ríos J, Castañeda J, Calle G, et al. Histerectomía laparoscópica total, curva de aprendizaje: experiencia de Clínica del Prado, Medellín, Colombia. Rev Chil Obstet Ginecol. 2010;75:367-74.

21. Perino A, Cucinella G, Venezia R, Castelli A, Cittadini E. Total laparoscopic hysterectomy versus total abdominal hysterectomy: an assessment of the learning curve in a prospective randomized study. Hum Reprod. 1999;14:2996-9.

22. Brummer TH, Seppala TT, Harkki PS. National learning curve for laparoscopic hysterectomy and trends in hysterectomy in Finland 2000-2005. Hum Reprod. 2008;23:840-5.

23. Mäkinen J, Johansson J, Tomás $C$, Tomás E, Heinonen PK, Laatikainen T, et al. Morbidity of 10110 hysterectomies by type of approach. Hum Reprod. 2001;16:1473-8.

24. Hopper AN, Jamison MH, Lewis WG. Learning curves in surgical practice. Postgrad Med J. 2007;83:777-9.

25. Pareja R, Nick AM, Schmeler KM, Frumovitz M, Soliman PT, Buitrago CA, et al. Quality of laparoscopic radical hysterectomy in developing countries: a comparison of surgical and oncologic outcomes between a comprehensive cancer center in the United States and a cancer center in Colombia. Gynecol Oncol. 2012;125:326-9.

26. Obermair A, Gebski V, Frumovitz M, Soliman PT, Schmeler KM, Levenback $C$, et al. A phase III randomized clinical trial comparing laparoscopic or robotic radical hysterectomy with abdominal radical hysterectomy in patients with early stage cervical cancer. J Minim Invasive Gynecol. 2008;15:584-8. 